\title{
Lipoma of the Parotid Gland
}

\author{
Kimberly N. Tong ${ }^{1} \cdot$ Sharon Seltzer ${ }^{2} \cdot$ James T. Castle $^{1} \mathbb{D}$
}

Received: 29 November 2018 / Accepted: 8 February 2019 / Published online: 19 March 2019

(c) This is a U.S. Government work and not under copyright protection in the US; foreign copyright protection may apply 2019

Keywords Lipoma $\cdot$ Parotid gland $\cdot$ Otolaryngology $\cdot$ Neoplasms-adipose tissue $\cdot$ Magnetic resonance imaging

\section{History}

A 46 year-old woman was referred to Otolaryngology for evaluation of a right-sided cheek swelling found at a routine physical. The patient was asymptomatic and without systemic disease. On clinical examination, she was noted to have a soft, $3 \mathrm{~cm}$ mobile mass arising from the region of the right parotid gland. Her facial nerve was fully intact and she had normal salivary flow through Stenson's duct. Utilizing magnetic resonance imaging (MRI) for further evaluation revealed a lesion with characteristics most consistent with a non-aggressive adipocytic tumor such as a lipoma. A right superficial parotidectomy was performed resulting in successful removal of the neoplasm without complication.

Disclaimer The views expressed in this manuscript are those of the authors and do not reflect the official policy of the Department of Navy, Air Force, Department of Defense, or U.S. Government. I certify that all individuals who qualify as authors have been listed; each has participated in the conception and design of this work, the writing of the document, and the approval of the submission of this version; that the document represents valid work; that if we used information derived from another source, we obtained all necessary approvals to use it and made appropriate acknowledgements in the document; and that each takes public responsibility for it. We are military Service Members. This work was prepared as part of our official duties. Title 17 U.S.C. 105 provides that 'Copyright protection under this title is not available for any work of the United States Government.' Title 17 U.S.C. 101 defines a United States Government work as a work prepared by a military Service Member or employee of the United States Government as part of that person's official duties.

James T. Castle

James.T.Castle4.mil@mail.mil

Kimberly N. Tong

Kimberly.N.Tong.mil@mail.mil

Sharon Seltzer

Sharon.Seltzer1.civ@mail.mil

\section{Imaging Features}

Multiplanar, multisequence images of the facial bones to include parotid glands were acquired on a $1.5 \mathrm{~T}$ General Electric scanner before (preGad) and after (postGad) the administration of intravenous gadolinium contrast. Sequences included axial and coronal T1-weighted (Ax T1, Cor T1), fat-suppressed T1 (T1 FS), fat suppressed axial T2-weighted (Ax T2 FS), and T2-weighted inversion recovery (STIR). At the lateral aspect of the right parotid gland within the parotid capsule a well-defined $1.3 \times 2.1 \times 3.2 \mathrm{~cm}$ mass noted. With respect to the parotid tissue, the mass was hyperintense on preGad T1 (Fig. 1a, b), hypointense on fat-suppressed T2 and STIR sequences (Fig. 2a, b), and followed subcutaneous fat signal on all imaging sequences.

After the administration of contrast, the mass was hypointense to parotid, homogeneous in signal with thin septations, and uniformly non-enhancing. It appeared encapsulated without invasion, edema, or differential enhancement at the interface with subcutaneous fat or adjacent parotid gland. Given the MRI tissue imaging characteristics, as well as demographics and clinical presentation, a nonaggressive adipocytic tumor, particularly classic lipoma, was favored.

\section{Diagnosis}

A superficial parotidectomy specimen measuring $6.8 \times 2.7 \times 2.0 \mathrm{~cm}$ was received. The specimen consisted of a rubbery mass of tan, multinodular soft tissue showing

1 Department of Oral \& Maxillofacial Pathology, Naval Postgraduate Dental School, Naval Medical Professional Development Center, 8955 Wood Road, Bethesda, MD 20889-5638, USA

2 Department of Radiology, Fort Belvoir Community Hospital, 9300 DeWitt Loop, Fort Belvoir, VA 22060, USA 


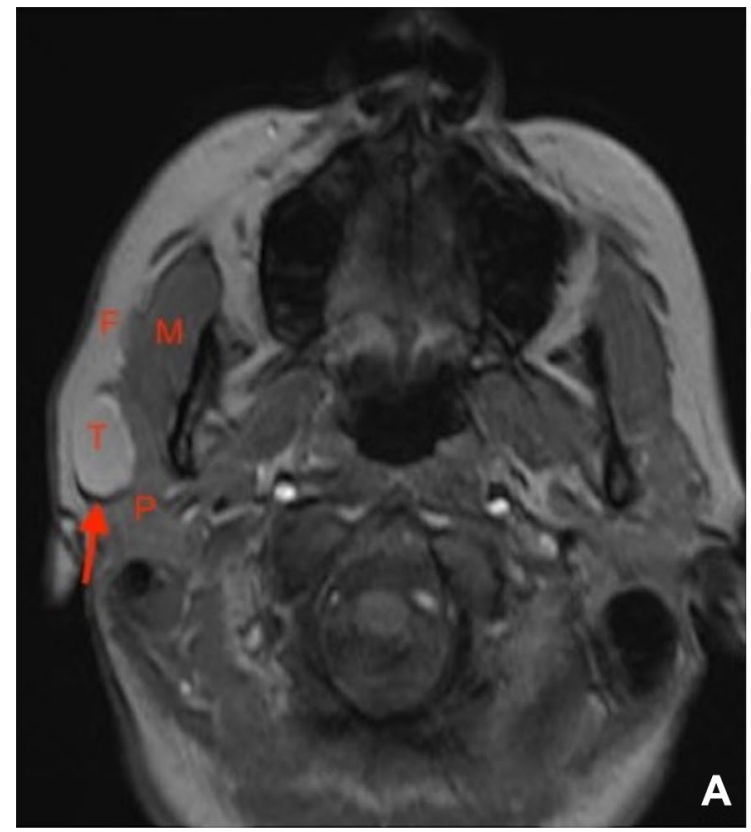

Fig. 1 a PreGad Axial T1: bright lesion (T), hyperintense to parotid (P) tissue and muscle (M), deep and isointense to subcutaneous fat (F). A T1 intermediate to dark capsule (arrow), best seen posteriorly, may represent both the parotid and the lipoma capsules abutting each

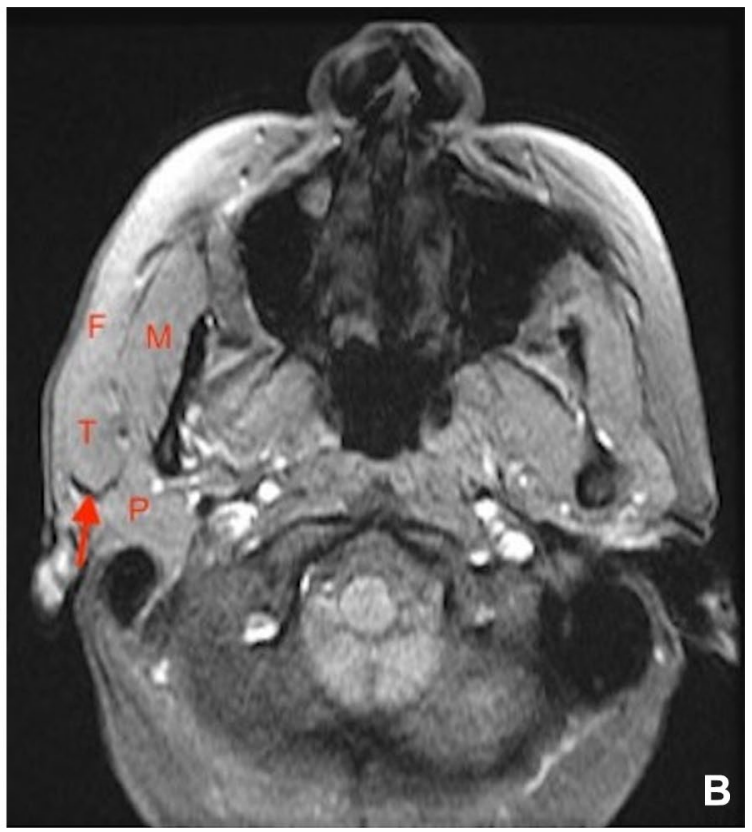

other. b PreGad Axial T1 FS highlighting the lesion (T) which is now isointense to parotid $(\mathrm{P})$ and muscle $(\mathrm{M})$ and isointense to subcutaneous fat $(\mathrm{F})$. Capsule is denoted by arrow

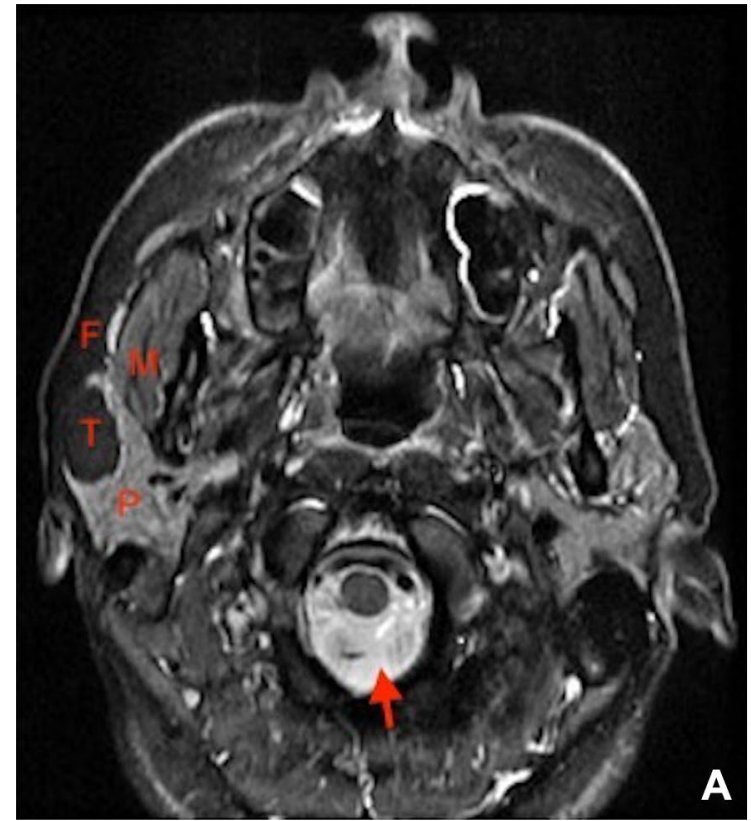

Fig. 2 a Axial STIR, essentially fat suppressed T2, where lesion (T) is T2 dark compared with cerebrospinal fluid CSF (arrow), isointense to subcutaneous fat $(\mathrm{F})$ and hypointense to parotid $(\mathrm{P})$ and to

flecks of peripherally placed yellow soft tissue interpreted as adipose tissue. Sectioning revealed an ovoid, well-circumscribed area showing a yellow cut surface consistent with adipose tissue along with a peripheral rim of fibrous

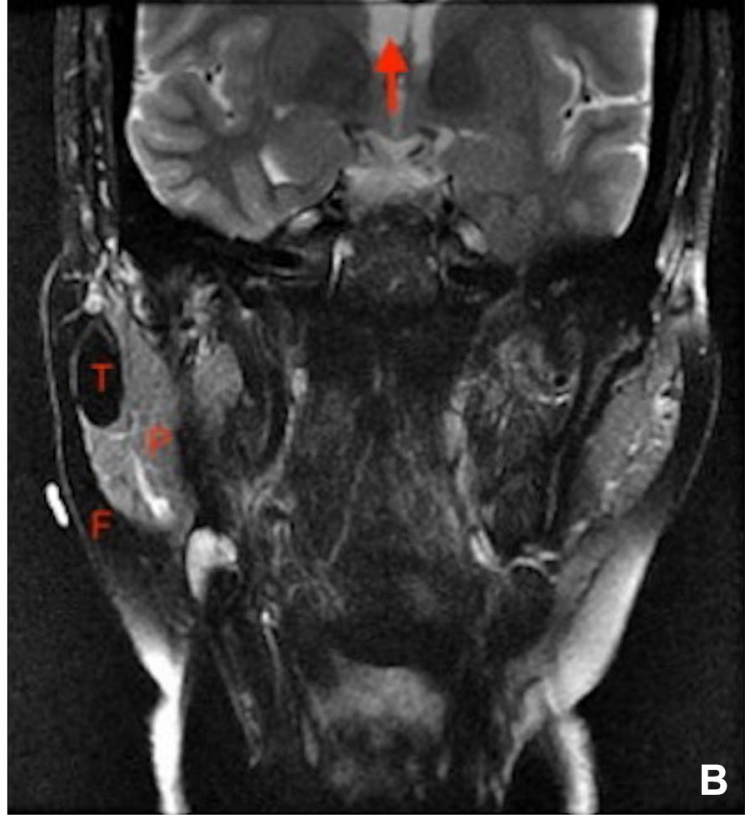

muscle (M). b Coronal T2 FS. The lesion (T) is T2 dark compared with intracranial CSF (arrow), isointense to subcutaneous fat $(\mathrm{F})$ and hypointense to parotid $(\mathrm{P})$ and to muscle $(\mathrm{M})$

connective tissue. This yellow component measured $2.3 \times 1.4 \times 1.0 \mathrm{~cm}$ in total. The area peripheral to the yellow component, best identified as unaffected parotid gland, consisted of a glistening red-tan mass of soft tissue most 
notable for numerous discrete lobules. Microscopic examination revealed a well-circumscribed aggregate of mature adipocytes that formed a large, well-demarcated mass composed of relatively equal sized adipocytes surrounded by a thin fibrous capsule of dense hyalinized fibrous connective and a rim of unremarkable serous salivary gland tissue (Fig. 3). In total, the findings supported the diagnosis of an intraparotid lipoma.

\section{Discussion}

While lipomas are the most commonly diagnosed soft tissue tumors in the body [1], lipomas of the parotid salivary gland are relatively rare comprising only $0.6-4.4 \%$ of reported benign parotid neoplasms [2]. Parotid lipomas often present as slow-growing asymptomatic nodular masses, with the majority involving only the superficial lobe [1-3]. Without additional preoperative studies, this benign clinical presentation is most often mistaken for Warthin tumor or pleomorphic adenoma $[1,3,4]$.

Potential preoperative imaging studies that may be used to further characterize a parotid soft tissue mass includes ultrasonography (US), magnetic resonance imaging (MRI) and computed tomography (CT). On US, head and neck lipomas are typically well-defined masses, hyperechoic to adjacent muscle and containing linear echogenic lines at right angles [4]. However, lipomas have also been described as isoechoic or hypoechoic, making US less specific than other imaging methods [3]. MRI is the preferred method for visualizing soft tissue neoplasms, providing the best soft tissue definition and often allowing visualization of the tumor capsule from surrounding adipose tissue [1]. Lipomas demonstrate high signal on T1-weighted images, low signal on

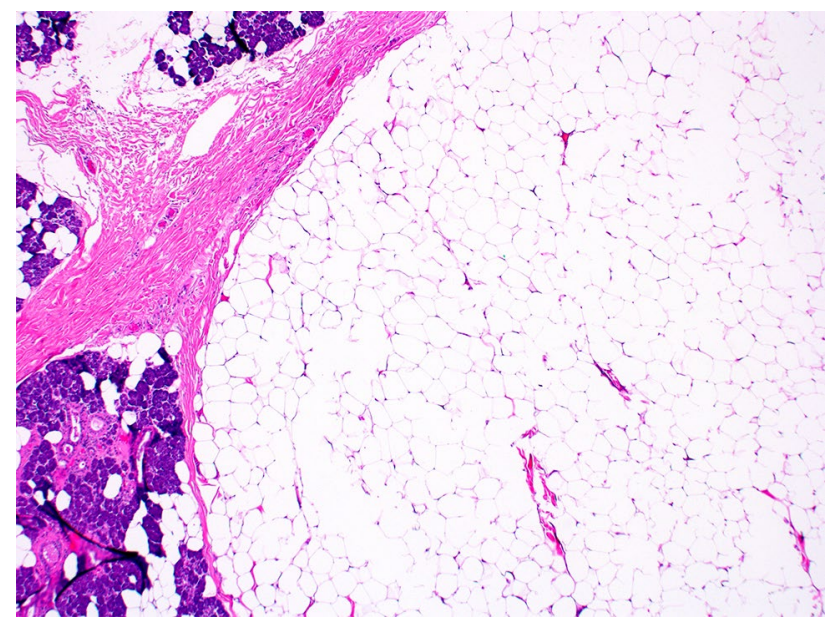

Fig. 3 A mass of mature, uniform adipocytes contained within parotid parenchyma is seen surrounded by a thin fibrous capsule
T2-weighted images and can be definitively diagnosed as tumors of adipocytic origin on fat-suppressed T1-weighted MRI sequences [1, 3-5]. The most common benign and malignant parotid neoplasms have different MRI tissue characteristics: benign and malignant pleomorphic adenomas and mucoepidermoid carcinoma tumors are variably $\mathrm{T} 2$ hyperintense and enhance, Warthin tumor is $\mathrm{T} 2$ hypointense, oncocytoma is $\mathrm{T} 1$ hypointense and hemangioma is variable in signal but is most often seen in the pediatric population.

Computed tomography is just as effective as MRI, if not more straightforward and a less costly option to aid in the diagnosis of a lipoma [3]. Being the only soft tissue with a density less than water, the CT attenuation ranges of -150 to -50 Hounsfield units are considered diagnostic for fat by most investigators $[1,3,5]$. However, CT does expose the patient to ionizing radiation, which could be avoided with MRI [1]. Imaging as a whole is limited in its inability to definitively distinguish benign from malignant, a distinction that can only be determined with histopathologic examination of the lesion [3, 4].

Parotid lipomas can further be classified based on location as well as histologic subtype with the designation being periparotid if they are found to be compressing the lateral surface of the parotid gland, and intraparotid when they are surrounded entirely by salivary gland tissue [3, 4]. A true lipoma is histopathologically characterized through identification of a thin fibrous capsule surrounding a neoplasm of mature, similarly-sized adipocytes. Identification of a capsule may aid in distinguishing such a neoplasm from pseudolipoma, lobular lipomatous atrophy, or lipomatosis, all of which are unencapsulated.

Pseudolipomas are surmised to be the result of a posttraumatic incident where prolapse of adipose tissue through fascia is induced by direct impact [6]. It is additionally speculated that pseudolipomas follow fat necrosis as a trigger which affects adipocytes by setting off a cascade of local inflammation resulting in hematoma formation, thus promoting formation of a lipoma [7]. Lobular lipomatous atrophy is usually associated with obstructive or inflammatory diseases of salivary glandular tissue and shows the affected lobule to be contiguous with other parenchymal salivary gland lobules that lack a capsule [8]. Histologic features of atrophic changes such as loss of glandular parenchyma, presence of thick-walled vessels and ductal hyperplasia with periductal fibrosis, characterize the findings.

Lipomatosis may be a bilateral and diffusely infiltrative, although the process resulting in a clinical swelling of the gland is exceedingly uncommon [8]. It is histologically characterized as fatty infiltration of the glandular parenchyma that is distributed in a sheet-like or random pattern [1,2,5, 6]. Although lipomatosis may be present as an idiopathic finding, it has been reported to be associated with hormonal 
and/or metabolic abnormalities, chronic alcoholism, malnutrition or drug therapy [5].

While the majority of parotid lipomas are of the classic lipoma subtype, other lipoma subtypes including spindle cell lipoma and hybrid lipoepithelial neoplasms such as nononcocytic sialolipomas and oncocytic lipoadenomas have been described in the literature [8-10]. Spindle cell lipomas are characterized by a background of prominent myxoid change, often with pronounced ropey collagen and mast cells amongst mature adipocytes. Sialolipomas most closely resemble normal salivary gland, but are distinguished by being clearly encapsulated neoplasms. They are found to be characteristically lobulated, biphasic tumors that are nononcocytic with evenly distributed salivary glandular tissue that is not neoplastic contained amongst abundant mature adipocytes. The fatty component is the dominant feature over the epithelial elements along with occasional foci of sebaceous metaplasia, variable periductal inflammation and fibrosis [8]. Oncocytic lipoadenomas are a lobulated finding that are predominantly composed of oncocytoma-like epithelial elements intermingled with variable amounts of adipocytic tissue, but consistently maintain a predominance of the epithelial component [8]. The oncocytic component may be scattered and intimately associated with individual fat cells or may form discrete nodules within the lipoma-like background. As in sialolipoma, sebaceous metaplasia, variable periductal inflammation and fibrosis may be present.

Although rarely reported as primary or metastatic parotid masses, clinicians still need to possess some amount of suspicion for malignant lipomatous tumors. These tumors do not present with a radiographic profile that distinguishes benign from malignant fat, although tumors such as pleomorphic, dedifferentiated and myxoid liposarcoma subtypes, among others, may show features of heterogeneous density on radiologic studies which is a departure from normally identified characteristics as previously mentioned [1].

Lipomas should be considered along with common salivary gland tumors in the differential diagnosis of slowgrowing asymptomatic soft tissue tumors of the parotid. CT and MRI can accurately characterize soft tissue neoplasms of adipocytic origin and should be utilized to define the size, location, and extent of the neoplasms prior to treatment. Definitive diagnosis can only be accomplished with histopathologic review. As part of that process, the myriad of histologic subtypes of classic lipoma should be recognized but, classification does not impact treatment or prognosis.

\section{Compliance with Ethical Standards}

Conflict of interest The authors declare that they have no conflict of interest.

Research Involving Human and Animal Participants This article does not contain any studies with human participants or animals performed by any of the authors.

\section{References}

1. Starkman SJ, Olsen SM, Lewis JE, et al. Lipomatous lesions of the parotid gland: analysis of 70 cases. Laryngoscope. 2013;123:651-6.

2. Houle A, Mandel L. Diagnosing the parotid lipoma: case report. NY State Dent J. 2015;81:48-50.

3. Abd El-Monem MH, Gaafar AH, Magdy EA. Lipomas of the head and neck: presentation variability and diagnostic work-up. $\mathrm{J}$ Laryngol Otol. 2006;120:47-55.

4. Husain N, Bandhauer F, Kurrer M, et al. Lipoma of the parotid gland. Neuroradiol J. 2008;21:81-6.

5. Ethunandan M, et al. Lipomatous lesions of the parotid gland. J Oral Maxillofac Surg. 2006;64:1583-6.

6. Sah K, Chandra S, Kale A, et al. Traumatic pseudolipoma causing facial asymmetry: an uncommon pathology and review of its pathogenesis. J Oral Maxillofac Pathol. 2011;15:113-15.

7. Copcu E, Sivrioglu NS. Posttraumatic lipoma: analysis of 10 cases and explanation of possible mechanisms. Dermatol Surg. 2003;28:215-20.

8. Agaimy A. Fat-containing salivary gland tumors: a review. Head Neck Pathol. 2013;7(Suppl 1):90-6.

9. Agaimy A, Ihrier S, Märkl B, et al. Lipomatous salivary gland tumors: a series of 31 cases spanning their morphological spectrum with emphasis on sialolipoma and oncocytic lipoadenoma. Am J Surg Pathol. 2013;37:128-37.

10. Furlong MA, Fanburg-Smith JC, Childers EL. Lipoma of the oral and maxillofacial region: site and subclassification of 125 cases. Oral Surg Oral Med Oral Pathol Oral Radiol Endodontol. 2004;98:441-50.

Publisher's Note Springer Nature remains neutral with regard to jurisdictional claims in published maps and institutional affiliations. 